\title{
Stimulus change and complexity in exploratory behavior
}

\author{
GEORGE T. TAYLOR \\ State University of New York, College at Potsdam, Potsdam, New York 13676
}

\begin{abstract}
It has recently been proposed (Fowler, 1967, 1971) that the incentive value of novelty can be defined in terms of stimulus change. An experiment is reported which examines the relationship between changes in complexity and the consequent exploratory behavior. Following extended exposure to a midcomplexity stimulus, rats were allowed to choose between a stimulus change and the now familiar midcomplexity stimulus. For some Ss the change was to a stimulus of higher complexity, and for others the change was to a lower complexity stimulus. The results suggest that physical stimulus changes to a stimulus of greater complexity is a more effective novel stimulus than physical stimulus changes to lesser complexity, although both changes may function as incentives.
\end{abstract}

One of the more enduring problems the reinforcement or incentive theorist must confront is to a priori identify those events that will serve as an incentive for a particular behavior (cf. Logan \& Wagner, 1965). This problem is particularly evident in the literature on exploratory behavior. The presumption of most authors (see Fowler, 1971) has been that "novel" stimuli have the capacity to reinforce, as well as energize, exploratory behavior.

Yet the concept of "novelty" has had diverse meanings. For some investigators, novelty is a change in illumination (Levin \& Forgays, 1959; Moon \& Lodahl, 1956; Williams, 1971). For others, it is the introduction of a checkerboard (Fowler, 1965), an elevated maze (Halliday, 1967), a floor insert containing various figurines (Wilson \& Taylor, 1969; Taylor, 1970), an opportunity to view another animal (Butler, 1954), or a variety of trinkets (Taylor, 1971).

Fowler $(1965,1967)$ has suggested that novelty functions as does any other more conventional incentive, such as food or water. Stimulus-change deprivation provides the antecedent conditions for the consequent exploration and stimulus change reinforces the exploratory behaviors. Fowler has thereby defined novelty in terms of stimulus change.

The main value of this analysis is that the incentive value of stimulus change is as quantifiable as are food or water incentives. For example, the greater the physical change in the stimulus, within limits, the greater the incentive value and the more powerful the effects upon exploratory behavior (Fowler, 1967).

However, stimulus change implies that stimulus increments and stimulus decrements of equal magnitudes should be equally effective. Consider an animal that has experienced a stimulus of a particular intensity for some period of time. A subsequent increase or decrease in stimulus intensity should serve as an incentive for exploration. Furthermore, the direction of the change should be irrelevant, for the incentive value should be relatively equal provided the two changes in intensity are physically identical.

Fowler (1967, p. 161) has recognized this logical extension of the stimulus change concept and cites one study in particular as suggesting that increments and decrements have comparable incentive effects. Moon and Lodahl (1956) demonstrated that monkeys would barpress to increase or decrease the illumination in a test apparatus. However, they failed to control the stimulus deprivation variable, and they gave only one short test session.

In the experiment reported here, rats were first given extended exposure to a midcomplexity stimulus. The animals were then allowed to choose between this familiar midcomplexity stimulus and a stimulus change. For some animals the change was to a stimulus of higher complexity, and for others the change was to a stimulus of lower complexity. The results bear directly upon the question of the role of complexity and stimulus change in the analysis of exploratory behavior.

\section{METHOD}

\section{Subjects}

The Ss were 48 male albino rats obtained from a commerical supplier. The animals, approximately 100 days old at the beginning of experimentation, were housed individually with water freely available. The animals were assigned randomly to one of six groups, $12 \mathrm{Ss}$ in each of the two experimental conditions and $6 \mathrm{Ss}$ in each of four control groups.

\section{Apparatus}

An unpainted wooden T-maze with a $1.29-\mathrm{m}$ runway and $.57-\mathrm{m}$ arms was used. The startbox measured $24 \times 11.6 \times 16.8 \mathrm{~cm}$ and the goalbox was $25 \times 11.6 \times 16.8 \mathrm{~cm}$. The floor of the apparatus was hardware cloth and the top was Plexiglas. Five doors, each $16.8 \times 11.6 \mathrm{~cm}$, were raised and lowered manually. The doors were located directly after the startbox, at the beginning of each of the arms, and immediately preceding each goal area.

The "novel" stimulus was one of four wooden floor inserts, representing three levels of complexity. Glued upon the rear surface of each insert were a number of small unpainted blocks of wood, $1 \times 1 \times 7.4 \mathrm{~cm}$, which served as novel "objects." The pair of identical midcomplexity inserts had 6 of the wooden objects, the low-complexity insert had 2, and the high-complexity insert had 10 objects. The inserts fit snugly into the entire goal area. Finally, each of the two goal areas housed a glass dish, $9 \times 2.2 \mathrm{~cm}$, containing five pellets of food. 
Table 1

The Two Complexity Stimuli Present as Alternatives in the T-Maze During the Various Stages

\begin{tabular}{lccc}
\hline Group & Stage I & Stage II & Stage III \\
\hline Exp A & Mid vs Mid & Mid vs High & Mid vs Low \\
Exp B & Mid vs Mid & Mid vs Low & Mid vs High \\
Control A & Mid vs Mid & Mid vs Mid & Mid vs High \\
Control B & Mid vs Mid & Mid vs Mid & Mid vs Low \\
Control C & Mid vs Mid & Mid vs High & Mid vs High \\
Control D & Mid vs Mid & Mid vs Low & Mid vs Low \\
\hline
\end{tabular}

\section{Procedure}

Pretraining. All Ss were gentled and adapted to a 23-h food-deprivation schedule for 7 days prior to the beginning of experimentation. The animals were magazine trained, without any of the inserts, in each of the arms for 2 pretraining days.

Overview. The experiment was divided into three stages, each 24 days in length. In each, the rats were given four spaced trials per day or a total of 96 trials in each stage. The animals were given two initial free-choice trials followed by two forced trials to the arm opposite that chosen on the free trials. The free-forced sequence insured equal experiences to each alternative (cf. Taylor, in press).

On each trial during the experiment, an animal was placed into the startbox and the start door raised. On a free-choice trial both arm doors were open and the animal was allowed to choose one alternative. If the trial was a forced one, the appropriate arm door remained closed and the animal was forced to run into the opened arm. To insure that the animal could not view the novel stimulus inserts until after the $S$ made his choice, the goal doors remained closed until the animal had made his way into the arm. The arm door was then lowered to capture the animal in that alternative. Quickly the goal door was raised and the $S$ was allowed to enter the goal area. The animal remained in the goalbox for $15 \mathrm{sec}$ and was removed to a retaining cage for approximately $2 \mathrm{~min}$ and the next trial.

Within each of the two alternatives was a stimulus insert of a particular complexity and five $45-\mathrm{mg}$ Noyes pellets. The pellets, identical numbers at each goal location, were used to insure a motivated animal throughout the rather lengthy stages of the experiment (see Knapp, Kause, \& Perkins, 1959). In each alternative, the food was located in a glass dish placed at the beginning of the insert. The novel stimulus was one of four floor inserts differing only in complexity, i.e., the number of wooden objects attached to the rear of the insert. A total of six groups, two experimental and four control conditions, were used. The experimental design within the various stages is represented in Table 1.

Stage I. All Ss were given identical experiences during the first stage. The midcomplexity inserts were located at each of the two alternatives. Each animal received free-choice and forced-choice trials to the two midcomplexity stimuli, i.e., the floor inserts containing six wooden objects each. Each $\mathrm{S}$ was given a total of 48 trials to each of the stimuli.

Stage II. Subsequently, the animals were divided into one of six groups, two experimental and four control conditions. One experimental group (Exp A) and one control group (Control C) were given free-forced trials to the midcomplexity stimulus and an incrementally changed complex stimulus, that is, a floor insert with 10 of the wooden objects. The second experimental group (Exp B) and one control group (Control D) were given trials to the midcomplexity stimulus and a physically comparable but decremental change in complexity of the stimulus insert, i.e., two objects on a floor insert. The position of the changed stimulus was counterbalanced within each group, so that the changed stimulus was always on the left for half of the Ss and always on the right for the other rats. Thus, an animal was given a choice to expose himself to the familiar stimulus or one changed in complexity. This change was upward for some animals and downward for others. Finally, the midcomplexity stimulus remained at both alternatives for Control Groups $A$ and B.

Stage III. The final stage involved reversing the complexity conditions for the experimental groups. Thus, the Exp A animals chose between the midcomplexity stimulus and, now, a stimulus of low complexity, that is, the two-object insert. The second experimental group, Exp B, now experienced the high-complexity insert at the changed stimulus locus. The control conditions corresponding to the two experimental groups (Control C and D) remained on the high- and low-complexity stimulus conditions, respectively. The other two control groups that had remained on the original midcomplexity inserts at both loci during both Stages I and II now experienced a changed stimulus at one alternative. One group (Control A) was given trials to either the midcomplexity insert or the high-complexity stimulus. The other group (Control B) experienced similar training, with the exception that the changed stimulus alternative was the low-complexity object.

\section{RESULTS}

The data of most interest were the two daily free-choice trials of the three stages. The first stage served to equate stimulus exposures for all Ss. The results were that the animals eventually evolved into a high incidence of alternation behavior between the two identical stimulus alternatives, i.e., midcomplexity stimuli, on the free-choice trials. Those data, as well as the control conditions remaining on the two midcomplexity stimuli during Stage II, were not graphed.

Instead, the percentages of turns toward the changed stimulus were averaged for the four groups experiencing a stimulus change in Stage II and all six groups in Stage III (see Fig. 1). Also, since the counterbalanced subgroups in each condition did not differ in any stage, the results of all the animals in each group were combined for the data analyses.

\section{Stage II}

The purpose of the second stage was to examine the effects of stimulus changes on subsequent choice behavior-changes which were comparable in magnitude but differing in direction. The suggestion from Fig. 1 is of an increasingly higher percentage of choices to the changed stimulus which then decreases only after extended experiences with that stimulus. Moreover, there is the indication that the rats with an incremental change (Exp A and Control C) exhibited much higher percentages of "novel" choices than Ss with a decremental stimulus change (Exp B and Control D).

An analysis of variance for the total numbers of choices to the changed stimulus was performed. The three conditions in the analyses were the $18 \mathrm{Ss}$ experiencing the incremental stimulus change, the $18 \mathrm{Ss}$ with a decremental change, and the 12 control animals remaining on the original task. The results indicated a significant value $[F(2,45)=51.30, p<.01]$. Subsequent a posteriori comparisons indicated that every condition differed from every other condition. Therefore, a stimulus change was capable of reinforcing choice 


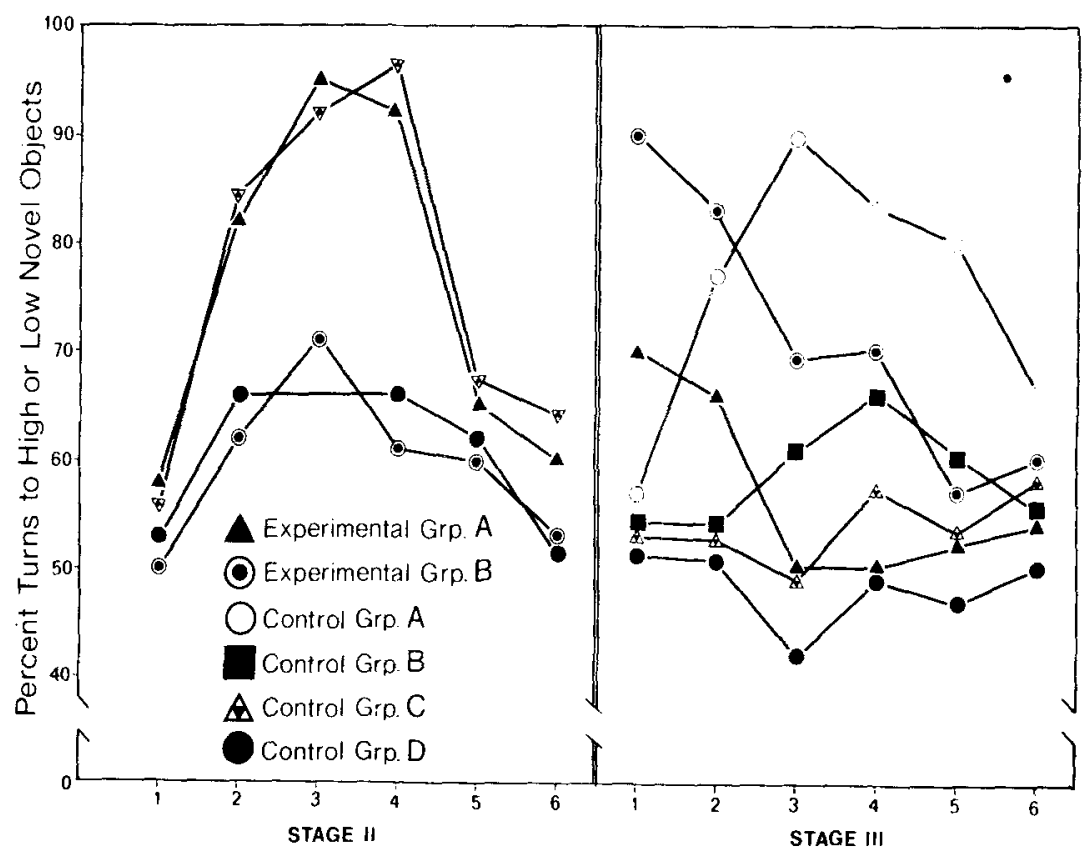

Fig. 1. The percentage of choices to the changed stimulus.

BLOCKS OF FOUR DAYS

behavior, yet an upward change in stimulus complexity was significantly more effective than was a physically comparable but downward stimulus change.

\section{Stage III}

The third stage was a bit more complex than the first two stages, for all six groups received different training. One purpose of the final stage was to reverse the changed complexity inserts for the two experimental groups. The reversal was to determine if there would be an appropriate reversal in preference behavior. As a control, some animals (Control C and D) were not reversed but remained on the high- or low-complexity conditions, respectively.

The figure suggests a reversal in preference behavior by the experimental groups when the complexity conditions were reversed. The $\operatorname{Exp~B}$ animals quickly increased the number of turns to the changed and, now, high-complexity stimulus. The second experimental group, Exp A, made an initial rise, although not to the level of the $\operatorname{Exp} B$ group, in choices to the changed but, now, low-complexity stimulus. Finally, both experimental groups appeared to choose the changed side more frequently than the control Ss remaining on the originally changed conditions.

Another purpose of the third stage was to examine longer lengths of stimulus-change deprivation and its effect upon a change in stimulus complexity. Two groups which had received extended training on the midcomplexity inserts (Control A and B) throughout both Stages I and II were given a changed stimulus at one locus. Control A, experiencing a highly complex stimulus, and Control B, confronted with a low-complexity insert, appeared to prefer the changed alternative. However, there is the suggestion that the incremental stimulus change was again more effective than the comparable decremental change.

Statistical support for these observations came from the application of an analysis of variance on the total numbers of choices to the changed stimulus in Stage III, with all six groups represented. The overall treatment effect was significant $[F(2,45)=31.47, p<.01]$. The a posteriori comparisons indicated that the two high-complexity conditions, Exp B and Control A, did not differ, yet both chose the changed stimulus more often than the other groups. However, the groups experiencing a decremental change, Exp A and Control B, were also superior to the control animals remaining on the low-complexity condition of Stage II (Control D).

Subsequently, two $t$ tests of Stage III preference behaviors were performed to determine if the longer periods of stimulus-change deprivation increased the attractiveness of the changed stimulus. A t test between Exp A, which had the high-complexity stimulus presented after 96 trials, and the Control A Ss, presented the same high-complexity insert after 192 trials, yielded a nonsignificant value $(t<1)$. Similarly, a $t$ test between the Exp B and Control B, which experienced the decremental stimulus change after 96 or 192 trials, respectively, also yielded a nonsignificant value $(t<1)$. Thus, the extended periods of stimulus-change deprivation did not produce exploratory preference behaviors differing from those animals receiving less stimulus-change deprivation.

\section{DISCUSSION}

The findings of the present experiment suggest several conclusions pertaining to novelty and the resultant exploratory behavior. First, the results demonstrate that a physical stimulus change, whether incremental or decremental in nature, is an effective incentive for 
exploratory behavior. These data lend support to the proposal of Fowler $(1967,1971)$ that "novelty" can be effectively defined in terms of stimulus change. Moreover, stimulus change, and therefore novelty, can be as quantitatively represented in a priori terms as can the more traditional incentives, e.g., food or water.

Second, the present investigation controlled and equated the amount of stimulus-change deprivation, i.e., stimulus preexposure, of each animal and confirmed the earlier findings of Moon and Lodahl (1956). That is, physical changes in stimulus intensity, both increases and decreases, can serve as an incentive.

The data, however, suggest that complexity as well as stimulus change plays an important role in the determination of exploratory behavior. A physical stimulus change to a lesser complexity stimulus was less effective than a similar change to a stimulus of greater complexity. To be sure, Fowler (1967, p. 191) has recognized the importance of complexity, as well as surprise, incongruity, and asymmetry, in an analysis of stimulus change. However, he does not propose the relationship between complexity and stimulus change. The present data indicate the relationship to be the following: Physical stimulus changes toward greater complexity are more effective than physical stimulus changes toward lesser complexity, although both changes may function as incentives.

A recent experiment (Unikel, 1971) has suggested a similar conclusion. Human Ss observed a series of lights of a given complexity for $30 \mathrm{~min}$. They were then allowed to choose to continue observing the same level of complexity or a newly introduced level. For some Ss the level of complexity was below that of the original level, and for others the new complexity was greater. The results demonstrated that, although all Ss preferred to view the changed stimulus, the change to the greater complexities was more effective than similar changes of similar magnitude toward the lesser complexities. These data are, of course, highly consonant with those of the present investigation.

Other aspects of the present experiment are worthy of comment. Note the preference behavior of the control animals (Control $\mathbf{A}$ and $\mathrm{B}$ ) given extended preexposure to the two midcomplexity inserts through both Stages I and II. When they were switched to the high- or low-complexity stimuli in the third stage, both performed similarly to the animals (Exp A and B) preexposed to the midcomplexity insert for a shorter period, i.e., through only Stage I. The incentive motivational theory of Fowler (1971) predicts that longer periods of stimulus-change deprivation should increase subsequent exploration. Those increases in exploration did not occur in the present study. However, one may propose that the animals reached an asymptotic level of stimulus-change deprivation after the 96 trials of Stage I and continued deprivation had little effect. These findings do, however, suggest the need for further parametric study of the effects of stimulus-change deprivation upon subsequent exploration.
Also, one may note that the reversal conditions of the experimental Ss (Exp A and B) in Stage III were such that the rats actually were confronted with a greater stimulus change than they had experienced in the second stage. For example, in the second stage the Exp A Ss experienced a stimulus change from midcomplexity to high complexity. Yet in the reversal, the stimulus change was from high complexity to low complexity. Despite the greater change in the third stage, the experimental animals exhibited preference behaviors comparable to the performances under the lesser changes of Stage II. Perhaps the animals experienced some absolute level of satiation to the stimulus objects which concealed the effects of the greater stimulus change in Stage III. Again, the data suggest additional parametric work.

In conclusion, the data are generally consistent with the stimulus-change concept of novelty (Fowler, 1967, 1971). Animals will choose an alternative in which a stimulus change has occurred. Moreover, a physical stimulus change to a stimulus of greater complexity appears more effective as an incentive than the same physical change to a stimulus of lesser complexity. However, additional experimental study is suggested before a precise relationship among stimulus-change deprivation, increased and decreased changes in the complexity of a stimulus, and the resulting exploratory behavior can be stated.

\section{REFERENCES}

Butler, R. A. Incentive conditions which influence visual exploration. Journal of Experimental Psychology, 1954, 48, 19-23.

Fowler, H. Curiosity and exploratory behavior. New York: MacMillan, 1965.

Fowler, H. Satiation and curiosity: Constructs for a drive and incentive-motivational theory of exploration. In $\mathrm{K}$. W. Spence and J. T. Spence (Eds.), The psychology of learning and motivation. Vol. 1. New York: Academic Press, 1967 Pp. 157-227.

Fowler, H. Implications of sensory reinforcement. In R. Glaser (Ed.), The nature of reinforcement. New York: Academic Press, 1971. Pp. 151-195.

Halliday, M. S. Exploratory behavior in elevated and enclosed mazes. Quarterly Journal of Experimental Psychology, 1967, 19, 254-263.

Knapp, R. K., Kause, R. H., \& Perkins, C. C. Immediate vs delayed shock in T-maze performance. Journal of Experimental Psychology, 1959, 58, 357-362.

Levin, H., \& Forgays, D. G. Learning as a function of sensory stimulation of various intensities. Journal of Comparative \& Physiological Psychology, 1959, 52, 195-201.

Logan, F. A., \& Wagner, A. R. Reward and punishment. Boston: Allyn \& Bacon, 1965.

Moon, L. E., \& Lodahl, T. M. The reinforcing effect of changes in illumination on lever-pressing in the monkey. American Journal of Psychology, 1956, 69, 288-290.

Taylor, G. T. Variability of food reward presentation effects upon exploratory behavior. Paper presented at the meeting of the Rocky Mountain Psychological Association, Salt Lake City, Utah, May 1970.

Taylor, G. T. The incentive value of complexity. Psychonomic Science, 1971, 22, 143-144.

Taylor, $G$. $T$. The varied functions of punishment in differential instrumental conditioning. Journal of Experimental Psychology, 1974, in press.

Unikel, I. P. Effects of changes in stimulation upon preference for stimulus complexity. Journal of Experimental Psychology, $1971,88,246-250$.

Williams, D. I. Maze exploration in the rat under different levels of illumination. Animal Behavior, 1971, 19, 365-367.

Wilson, W. J., \& Taylor, G. T. Food and novel objects as incentives. Psychonomic Science, 1969, 14, 120-121. 